\title{
Criteria for identifying the molecular basis of the engram (CaMKII, PKMzeta)
}

\author{
John Lisman(D)
}

\begin{abstract}
The engram refers to the molecular changes by which a memory is stored in the brain. Substantial evidence suggests that memory involves learning-dependent changes at synapses, a process termed long-term potentiation (LTP). Thus, understanding the storages process that underlies LTP may provide insight into how the engram is stored. LTP involves induction, maintenance (storage), and expression sub-processes; special tests are required to specifically reveal properties of the storage process. The strongest of these is the Erasure test in which a transiently applied agent that attacks a putative storage molecule may lead to persistent erasure of previously induced LTP/memory. Two major hypotheses have been proposed for LTP/memory storage: the CaMKII and PKM-zeta hypotheses. After discussing the tests that can be used to identify the engram (Necessity test, Saturation/Occlusion test, Erasure test), the status of these hypotheses is evaluated, based on the literature on LTP and memory-guided behavior. Review of the literature indicates that all three tests noted above support the CaMKII hypothesis when done at both the LTP level and at the behavioral level. Taken together, the results strongly suggest that the engram is stored by an LTP process in which CaMKII is a critical memory storage molecule.
\end{abstract}

Keywords: Herpes simplex virus, K42 M, Place aversion, Occlusion, Memory

\section{Introduction}

During learning, our brains are modified in such a way that the learned information can later be recalled, even many years later. The molecular modifications that store that information form the engram. Those modifications are likely to be contained in only a subset of neurons, and recent experiments confirm this directly. The experiments that identify the neurons that store the engram take advantage of the fact that i $\mu \mu$ ediate early genes are turned on in the subset of neurons that are strongly activated during learning. By linking expression of channelrhodopsin to these genes, it has become possible to visualize and manipulate the activity of this subset. The key experimental result is that optogenetically exciting these cells elicits the behavior expected of memory recall [1]. It can therefore be concluded that the optogenetically excited cells either contain the engram or excite cells that do.

For the engram to mediate the recall process, the storage processes of the engram must affect neuronal signaling by an "expression process." This might occur by

Correspondence: lisman@brandeis.edu

Department of Biology, Brandeis University, Waltham, MA 02453, USA making the cells that contain the engram more excitable (e.g., by modifying intrinsic non-synaptic conductances); alternatively, it may occur through modifications of synaptic function. Since the discovery of long-term potentiation (LTP), an activity-dependent and long-lasting increase of synaptic strength, it has been suspected that the engram involves changes in synaptic signaling mediated by an LTP-like process $[2,3]$. The connection between LTP and memory is now supported by multiple lines of evidence [4-6] (but see [7]). Furthermore, LTP has been found to have properties that make it very well suited as a memory mechanism. First, analysis of LTP has shown that it enables the storage of vast amounts of information. Each of the over 10,000 synapses on a cell can be modified by LTP in a synapse-specific manner [8]. Gradations in synaptic strength vary over a 10 -fold range ( 3 bits of information) [9]. Therefore, if one considers just the CA3 region of the hippocampus, a region strongly implicated in episodic memory, the 3 million CA3 pyramidal cells in humans [10] contain about 30 billion synapses, thus making the storage of 100 billion bits of information possible. Second, LTP has been demonstrated [11] to have the Hebbian properties required 
to form meaningful associations in neuronal networks (LTP occurs at a synapse if there is both presynaptic activity and strong postsynaptic depolarization). Thus LTP has the desired properties to encode memory.

It follows that, in order to understand the molecular basis of the engram, it is important to identify the molecular processes responsible for the information storage that underlies the maintenance of LTP. When we consider how genetic memory is stored, the answer is rather simple: most genetic information is stored in the base sequences of DNA. It is natural to wonder whether the mechanisms responsible for storing the engram will be similarly simple. Because the criteria for identifying the biochemical basis of the engram have not been previously articulated, I will start by discussing appropriate criteria. I will then use these criteria to evaluate two major hypotheses for engram storage: the CaMKII (Calcium-Calmodulin Protein Kinase type II) hypothesis $[12,13]$ and the PKM-zeta (Protein Kinase M - zeta) hypothesis $[14,15]$. Other hypotheses $[16,17]$ that have not received as much investigation will be not be discussed.

\section{Induction, maintenance, and expression processes that underlie late LTP}

After LTP is induced, a variety of presynaptic and postsynaptic changes can produce short-lasting changes in synaptic transmission. Some of these may last only for seconds, but even weak induction protocols produce potentiation that can last for many minutes. The potentiation evident during the first $30 \mathrm{~min}$ after induction is generally referred to as early LTP. If the induction conditions are sufficiently strong, early LTP is followed by biochemically and structurally different processes that produce stable strengthening of the synapse; these processes are referred to as late LTP. Notably, protein synthesis inhibitors block late LTP, but not early LTP [18].

The processes that underlie the LTP can be classified into three functionally different categories: induction, maintenance, and expression processes (Fig. 1). Induction refers to events that occur near the time of stimulation and that trigger the downstream maintenance and expression processes. For example, because late LTP requires protein synthesis, the mechanisms that turn on this synthesis would be considered induction processes. The maintenance process is what underlies storage of the engram. Finally, via expression processes, the maintenance process leads to potentiation of the current through the AMPA (Alpha-amino-3-hydoxy-5-methyl-4isoxazolepropionic acid) type of glutamate-activated ion channels, thereby leading to the observed potentiation of the EPSP (excitatory postsynaptic potentials). Expression processes could in principle be simple. For instance, if memory maintenance was due to the amount of activated kinase at the synapse, expression could simply be the phosphorylation of AMPA channels by the kinase. On the other hand, expression could be more complex and could involve a kinase-initiated cascade that leads to enhanced AMPA transmission through multiple steps. The cascade might work to enhance delivery of channels to the synapse and/or to enhance the number of structural slots capable of anchoring the channels at the synapse. Indeed, given the evidence that late LTP involves structural enlargement of the synapse $[19,20]$, it would seem that expression mechanisms that couple storage process to structural changes must be present.

In searching for the molecular basis of the engram, it is key to identify the mechanisms that specifically underlie the LTP maintenance process. That said, less-specific tests can also be useful; notably, if knocking out a protein reveals that it is not necessary for LTP, then it certainly cannot be part of the maintenance process. For this reason, the following section discusses three types of tests (Necessary, Saturation/Occlusion, and Erasure tests) used to explore the role of molecules in LTP and learning, even though only the Erasure test is powerful enough to specifically identify a role of a molecule in memory maintenance.

\section{Experimental tests that distinguish induction, maintenance, and expression processes Necessary test}

A commonly used test for determining whether a molecule is involved in LTP is to pharmacologically inhibit a molecule or to genetically knock it out. If this has no

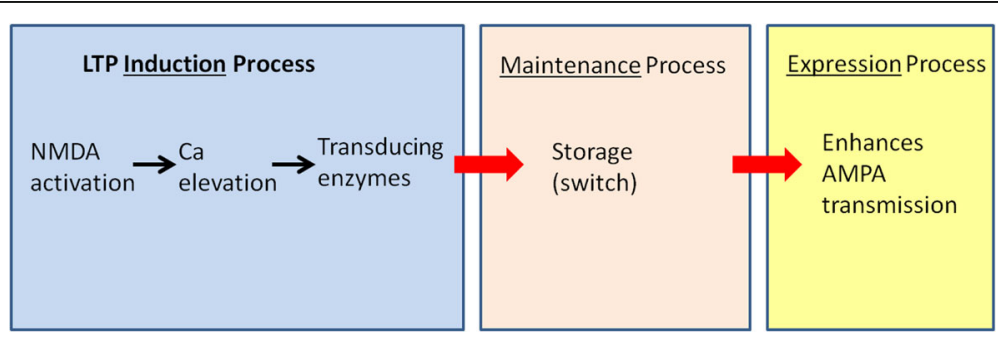

Fig. 1 Schematic of the three subprocesses in LTP. The engram is stored by the maintenance process and is specific for each of $\sim 10,000$ synapses in a neuron 
effect on LTP, then the molecule cannot be necessary for any LTP subprocess. If LTP is reduced or blocked, the molecule must have a role in one or more of the LTP subprocesses.

Some inhibitors have no effect on the earliest phases of LTP but block late LTP. It has been tempting to conclude that the targeted protein is therefore responsible for memory maintenance, but this is not a correct conclusion. The processes responsible for early and late LTP are biochemically very different, so finding an agent that selectively affects late LTP is not surprising given that the agent could be affecting events required for the induction of late LTP, but not early LTP (e.g., protein synthesis). Thus selective effects on late LTP do not imply a role of a target protein in the maintenance of late LTP. For this reason, the Necessary test can rule out the role of a protein in storing the engram but cannot provide positive evidence for such a role.

\section{Saturation/occlusion test}

In this test, an activated form of a protein is introduced into a neuron and the resulting change in the synaptic response is measured. If the protein enhances AMPARmediated transmission, it may or may not do so by the same biochemical process that occurs during LTP. This same/not-same issue can be investigated by studying the interaction of the two forms of potentiation. For instance, this can be done by producing potentiation with activated protein and then delivering a saturating LTP induction protocol (it is known that synaptic strength can be saturated). If the activated protein is indeed part of the normal LTP transduction pathway, subsequent delivery of an LTP induction protocol should have no effect (or at least a smaller effect than normal). Alternatively, subsequently normal LTP induction would indicate that the potentiation mechanism utilized by the protein was not the same potentiation mechanism that occurs during LTP. A putative engram molecule must pass this test. However, any molecule necessary for the induction, maintenance, or expression process can pass this test. Thus this test does not provide specific information about the storage mechanism. Nevertheless, the test is useful because it can rule out proteins that potentiate transmission by a process different from that which occurs during LTP/memory.

\section{Erasure test}

This is the sole test that is powerful enough to prove that a molecule is involved in storage of the engram. In this test, LTP is induced. Later, some kind of pharmacological or genetically expressed agent is used to attack the putative memory molecule. One then determines whether this reduces LTP. Since the agent was applied after LTP induction, any observed reduction cannot be due to an effect on induction processes. This reduction must be due to either an effect on maintenance or expression processes; removal of the agent can determine which is the case. If an expression process was affected, the remaining maintenance process will restore LTP. On the other hand, if the engram itself was destroyed, LTP will not recover (i.e., erasure occurred). One caveat, however, remains: a persistent reduction in LTP might be due to damage to the cell rather than erasure. It is thus critical to rule this out by showing that LTP can be reinduced. If this can be accomplished, it rules out damage of learning and recall processes and indicates that the memory maintenance process was indeed erased, not simply damaged. If a hypothesis passes this form of the Erasure test, it is appropriate to conclude that engram "erasure" occurred and that the protein being targeted is a required molecular component of the engram.

Unfortunately, proper conduct of the erasure test poses a technical difficulty. As noted above, it is crucial that the agent used to attack the putative memory molecule be applied and then removed before further testing. If the agent is not removed, a decrease in LTP or memory could be attributed to effects on the expression process rather than the maintenance process. Thus, identifying the molecular basis of the engram requires a method that allows an agent to be both applied and then removed before subsequent testing, a requirement not easily met when molecularly specific genetic methods are utilized.

\section{Using the above tests to evaluate the PKM-zeta model}

PKM-zeta is a constitutively active type of atypical protein kinase $\mathrm{C}$. It is synthesized for long periods after LTP induction [21]. It became a particularly promising engram candidate because a peptide inhibitor of this kinase, ZIP, produced powerful interference with LTP and memory maintenance in a variety of systems [22].

\section{Necessary test}

The concentration of ZIP used in in vivo experiments was several orders of magnitude greater than needed in slice experiments, raising questions about specificity [23]. Thus, confirmation of the PKM-zeta hypothesis with more specific genetic tools has been desirable. The first genetic experiments showed that PKM-zeta failed the Necessary test: knockout of PKM-zeta had little effect on LTP or memory $[24,25]$. Moreover, the electrophysiological effects of ZIP were still seen in the knockout, indicating off-target effects. These results appeared to rule out a simple PKM-zeta hypothesis. Fortunately, more specific methods have now been brought to bear on the problem. Recent work has used genetically based antisense or dominant-negative approaches [15]. Using these methods, it was found that inhibition of 
PKM-zeta reduced late LTP and memory performance $[15,21,26]$. These molecular approaches are more specific than ZIP and leave little doubt that PKM-zeta has an important role in LTP. However, to account for all of the data, a more complex hypothesis is required. It has been suggested that, when PKM-zeta is knocked out, a related atypical Protein Kinase C (PKC-lambda) takes over [15]. Confirmation of this possibility awaits results with knockout of both kinases.

\section{Occlusion test}

Using a slice preparation, it was found that introduction of active PKM-zeta produces potentiation and that it then becomes impossible to produce LTP [27]. These results thus show the saturation/occlusion expected if PKM-zeta is important in LTP. However, quite different results have been obtained in two studies that used an in vivo approach and genetic overexpression of the enzyme. It was found that synaptic strength was increased (Fig. 2a) as expected but that LTP and memory were also increased, contrary to the prediction of occlusion (Fig. 2b, c) [28] (for similar effects see [26]). On the basis of this failure of occlusion, it was concluded that PKM-zeta is not critical for maintenance but is instead a modulator of LTP [28].

\section{Erasure test}

In an elegant application of the erasure test, it was shown that application of ZIP to the hippocampus in vivo could erase conditioned place avoidance [22]. This erasure persisted long after ZIP injection; it is therefore unlikely that erasure was due to the continued presence of ZIP. It thus appeared that PKM-zeta had passed the critical erasure test. However, recent work has identified major problems with the specificity of ZIP. When activity of endogenous PKM-zeta was measured in live cells, it was found that ZIP was an ineffective inhibitor [29]. Other work showed that ZIP can have toxic effects [30] and has powerful effects on processes other than synaptic transmission [31]. Taken together, these results make it difficult to use ZIP to determine the molecular basis of the engram.

New methods have been used to study PKM-zeta's role in LTP/memory based on improved pharmacological agents and genetic methods [15, 21]. However, none of these studies have yet conducted the Erasure test as outlined above (removal on the attacking substance), a requirement that was met in the study that originally provided strong support for the PKM-zeta hypothesis [22]. Notably, in a recent study, application of anti-sense PKM-zeta to the brain reduced the learning-dependent increase in PKM-zeta and reduced memory performance [21]. However, in the experiments of Fig. 3a, the antisense was present during learning and was probably still present during the testing of 1-day memory (virally expressed proteins are likely to persist for at least a day). Thus, these agents may well have affected induction and/or expression processes. This problem with the design of the Erasure test indicates that no firm conclusion can yet be reached about the role of PKM-zeta in the maintenance process.

\section{Using the above tests to evaluate the CaMKII model}

CaMKII is one of the most abundant brain proteins. It exists in the cytoplasm at high concentration but is further concentrated in the postsynaptic density of glutamatergic synapses where it is a major protein [32]. The kinase holoenzyme consists of two rings of six subunits, each of which is catalytic. When $\mathrm{Ca}^{2+}$ enters the synapse during LTP induction, it leads to efficient activation of the CaMKII within spines (reviewed in [33]). This activation produces autophosphorylation of T286 sites on the kinase, a phoshorylation that makes the kinase persistently active even after $\mathrm{Ca}^{2+}$ levels fall [34]. Most of the 1000 CaMKII molecules [35] within a spine are inactivated within minutes [36], but a small pool (on the order of 50) can bind to the PSD and persist there for at least an hour [37]. Recent work indicates that the PSD itself has two compartments, a core region directly juxtaposed

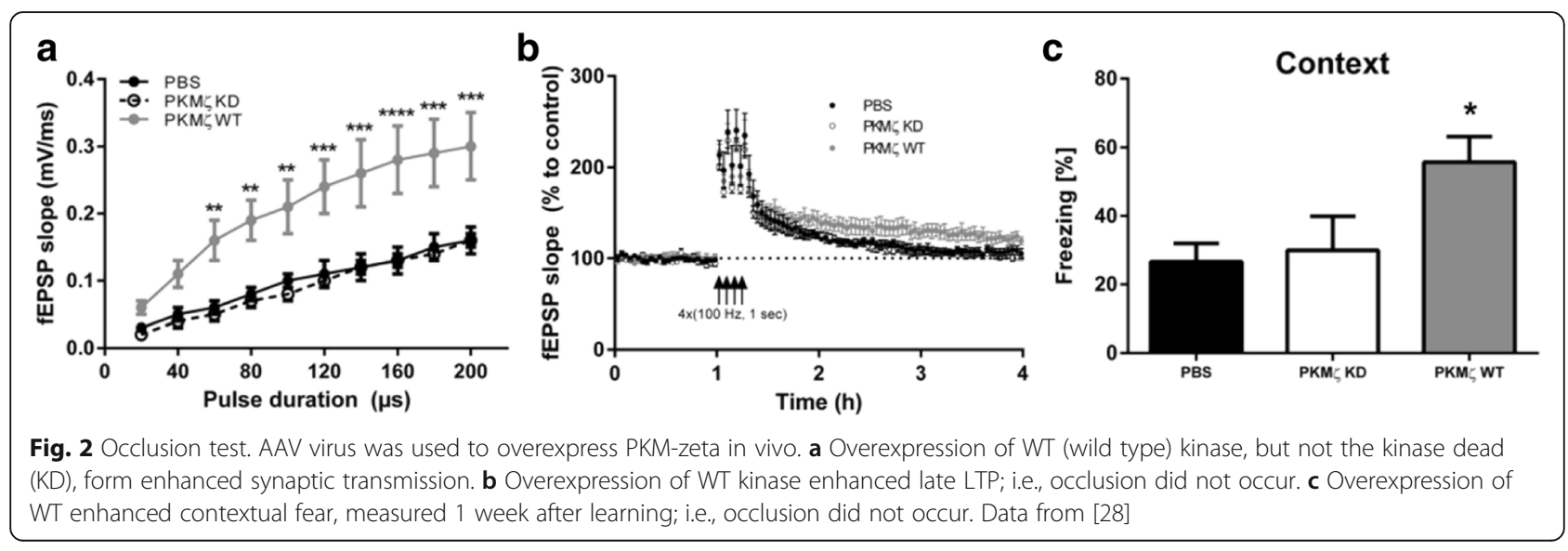




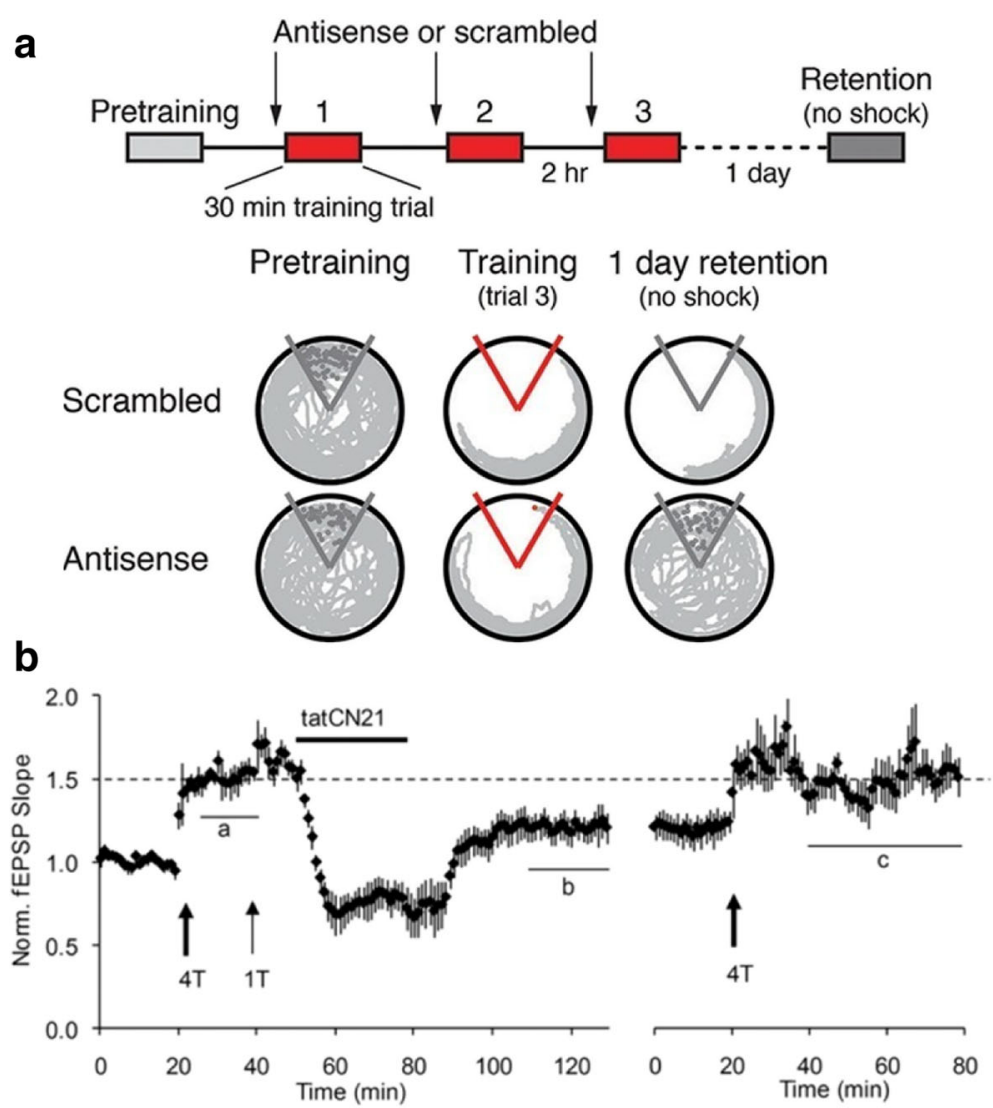

Fig. 3 Use of the erasure test. a (Top) Protocol for testing the effect of PKM-zeta antisense (injected into hippocampus) on 1-day memory. (Bottom) Grey lines show track of rat on rotating platform that moved the rat into triangular shock zone defined relative to the room. After injection of scrambled DNA, the rat learned to avoid the shock zone and remembered 1 day later. If antisense was injected into the brain during multiple phases of the learning process, 1-day retention was abolished. Because the antisense was present during learning and probably also during retention, the failure of memory may be due to effects on induction or expression processes and thus do not provide specific information about the maintenance process. From [15] b. (left) Maximal LTP was induced by 4 tetani delivered to the CA1 region of a hippocampal slice. Bath application of tatCN21, a peptide that interferes with CaMKII function, produced a decrease in response that persisted after removal of tatCN21. Erasure of LTP was confirmed by the fact that LTP could then be reinduced (right). From [50]

to the postsynaptic membrane and the more distant pallium region [38]. Most of the 50 or more CaMKII molecules in the PSD are in the pallium, but a few (on the order of 10) are in the core [39], where they may be bound to NMDARs $[40,41]$. There are reasons to think that it is this pool that is most important in LTP [42]. For a review of CaMKII function in LTP, see [33].

\section{Necessary test}

Knockout of CaMKII-alpha [43] or knockin of a mutant form that cannot autophosphorylate (T286A) [44] or is catalytically dead (K42 M) [45] greatly reduces LTP and memory. These results indicate that CaMKII must have a critical role in induction, maintenance, or expression processes. Studies of knockout and knockin mutations showed that the animals had strong deficits in memoryguided behavior, consistent with a critical role of LTP in memory.

\section{Occlusion/saturation test}

Intracellular application of the catalytic region of CaMKII potentiates transmission and strongly inhibits the induction of subsequent LTP [46]. Similar results were obtained by overexpression of activated CaMKII holoenzyme (T286D/T305A/T306A) [47]. These results thus suggest that activated CaMKII has powerful capability to potentiate AMPAR transmission and that this capability is utilized during the maintenance phase of LTP.

The occlusion/saturation test has been utilized to study behaviorally -defined memory and, specifically, the role of LTP in memory. In a critical set of experiments [4], learning occurred and was later followed by saturating induction of LTP in the dentate gyrus. This produced a strong deficit in subsequent memory behavior, as expected if saturation degraded memory. Stated differently, if memory depends on the differential strength of synapses, strengthening them all would be expected to degrade memory. 
Recent work has used a conceptually related strategy to test the role of CaMKII in memory-guided behavior [48]. In these experiments, animals first learned a conditioned place avoidance task. Several days later, a Herpes Simplex viral vector (HSV) was used to deliver activated CaMKII (T286D/T305A/T306A) to the hippocampus. Prior work had showed that this mutant strongly potentiates synapses, driving them to saturation, as indicated by the inability to induce further potentiation using strong synaptic stimulation [47]. When memory was tested at the time of strong expression of activated CaMKII (3 days after viral injection), memory behavior was strongly inhibited (Fig. 4). These results thus support the concept that memory is mediated by an LTP-like process dependent on CaMKII.

\section{Erasure test}

The erasure test for LTP was conducted by bath application of a peptide (TatCN21) that inhibits CaMKII and interferes with its binding to the NMDAR [49]. Transient application of the peptide after LTP induction reversed established LTP, which could then be reinduced by an LTP induction protocol (Fig. 3b) [50].

These results suggest that LTP erasure occurred, but several lines of other experiments strengthen this conclusion. First, the ability of $20 \mu \mathrm{M}$ of TatCN21 to interfere with the CaMKII/NMDAR complex in slices was confirmed biochemically [50]. Second, erasure produced by tatCN27, another CaMKIIN-derived peptide, was not simply due to LTD processes [51]. Third, the effect did not occur in neonatal animals that lack CaMKII-alpha in
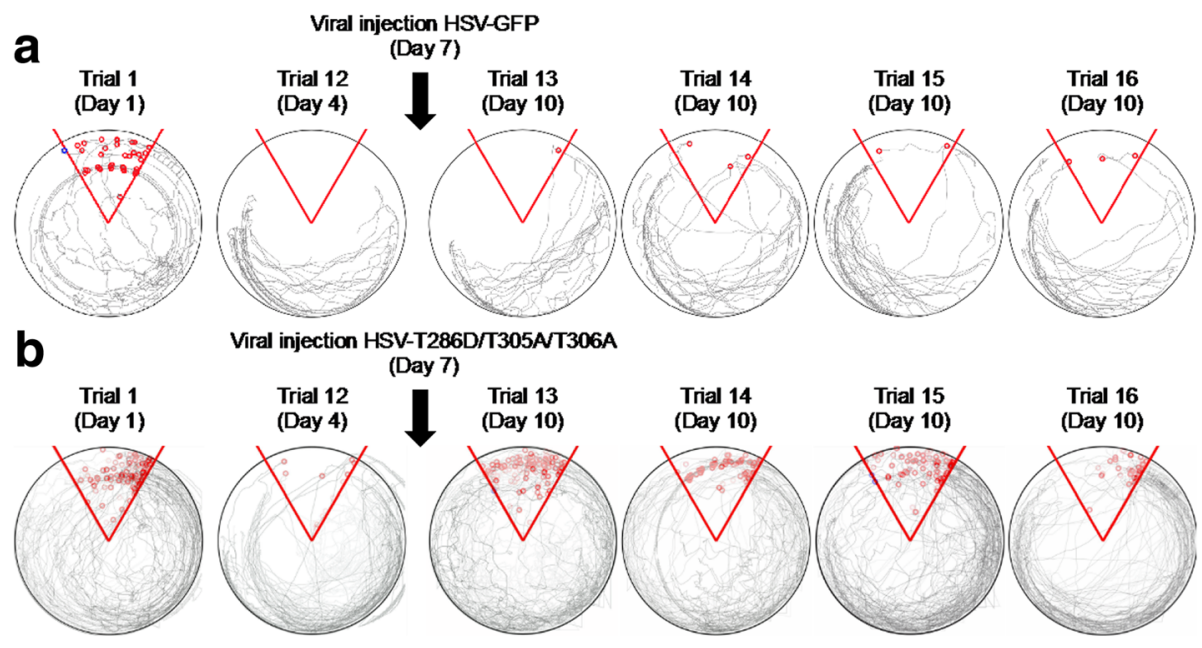

Viral injection HSV-T286D/T305AT306A
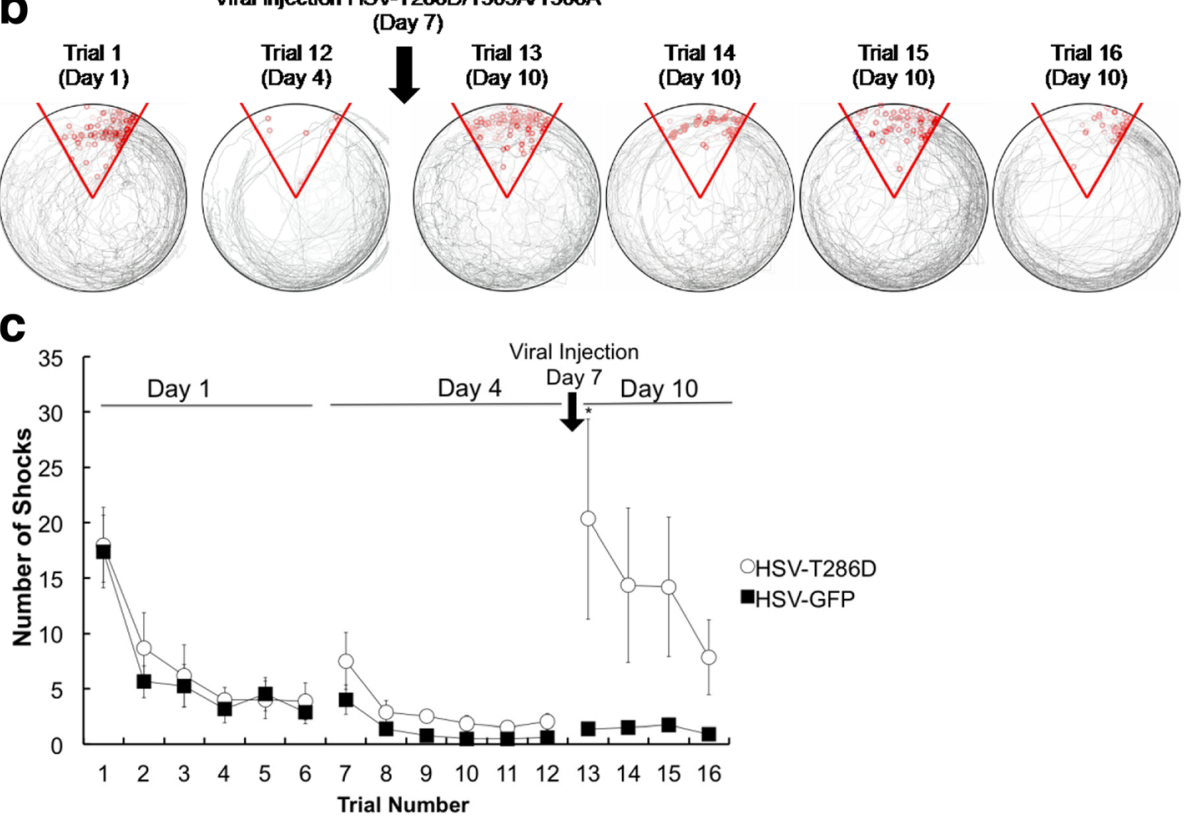

Fig. 4 Saturation test. a Path of rats (grey; superposition of six experiments) on circular platform before and after injection of control virus expressing GFP; shock zone (red pie shape) and individual shocks given (small red circles). Trial 1 is first training trial on day 1; trial 12 is last training trial (on day 4); reduction in number of shocks indicates learning. Trial 13 tests memory retention on day 10, 3 days after viral injection. Trials 14-16 show a deficit in relearning on day 10. b Path of rats before and after injection of virus expressing activated CaMKII (T286D/T305A/T306A). c Summary data. After viral expression of T286D/T305A/T306A, memory was poor $(p=0.02, \mathrm{D}=0.75)(n=6)$ compared to GFP controls. After viral expression of GFP, memory was strong in one group measured at day $10(n=2)$ and in another group measured at day $16(n=6)$ (the groups are not significantly different, so data is combined here as "day 10" $(n=8)$ ). For CaMKI**injected animals, memory on day 10 was not significantly different than memory on day 16 for K42 Minjected animals ( $p=0.8096, D=0.3333$ ). A two-sample $K-S$ test was used to determine statistical significance. Error bars represent mean $\pm S E M$. Asterisks indicate statistical significance ( $p<0.05$; Kolmogorov-Smirnov test). Reprinted with permission from [48] 
their PSDs [51]. Fourth, the erasure of LTP by tatCN21 was replicated [52] and demonstrated to be a postsynaptic effect. Fifth, it was shown that effects of tatCN21 were reduced in mice having a GluN2B mutation that interfered with the ability of CaMKII to bind to NMDARs [52]. These additional experiments, together with the primary results of Fig. 3b, make a strong case that CaMKII mediates the LTP storage process. For a full discussion of why other CaMKII inhibitors do not produce this effect seen in Fig. 3b, see [48] (briefly, only CN inhibitors at high concentration can interfere with the binding of CaMKII to GluN2B, NR2B NMDA receptor).

In the most critical test of the CaMKII in memory, the Erasure test was used to determine whether interfering with CaMKII could erase a behaviorally defined memory. In these experiments, a dominant-negative form of CaMKII was expressed several days after learning. A requirement of the Erasure test is that the dominant negative be only transiently expressed. Such transient expression is a well-established property of HSV [53], a virus chosen for this reason. Memory was tested 10 days after viral transfection, a time at which CaMKII expression was demonstrated to have ceased. As shown in
Fig. 5, memory was strongly reduced. Given that the dominant negative was no longer present, this effect is unlikely to involve expression processes. However, the reduction of memory might have resulted from damage to the hippocampus, but the fact that relearning could occur argues strongly against this. In an important further control, the same viral strategy was used to express wild-type CaMKII. In this case (the difference being only one amino acid), no erasure was produced. These results thus suggest that memory, like LTP, can be erased by interference with CaMKII function.

\section{Additional criteria \\ Mechanisms of stability}

A satisfactory molecular theory of memory storage needs to address the question of how the stability of memory is achieved. Memory lasts a long time compared to the lifetime of synaptic proteins, all of which undergo turnover within a week or less [54]. Thus, particular mechanisms must exist to ensure stable information storage by unstable molecules. Solutions to this problem have been proposed for both the PKM-zeta [55] and CaMKII models [12, 56] (Fig. 6; for explanations, see caption). At

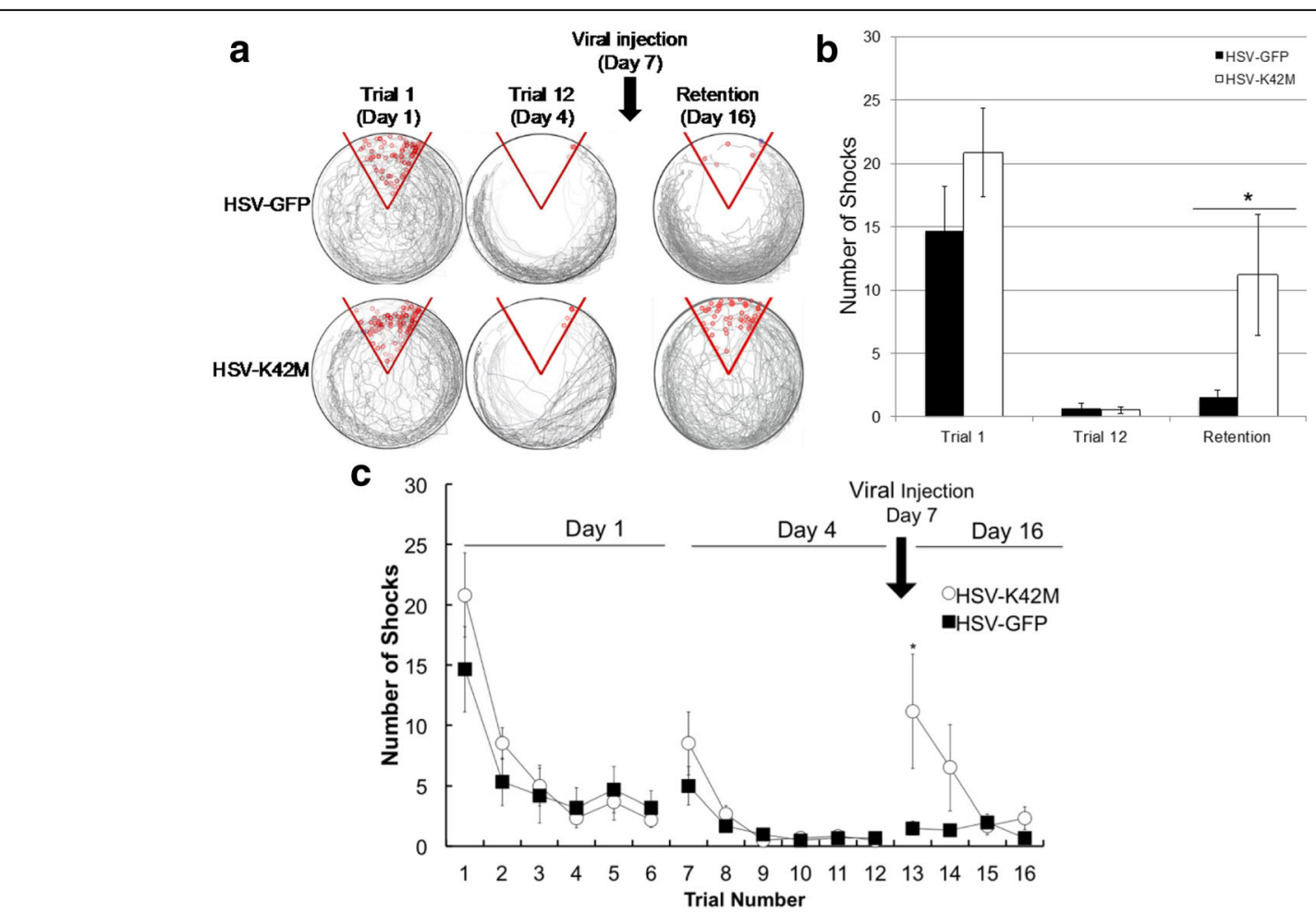

Fig. 5 Erasure test. Memory was tested 9 days after virus injection (day 16), a time at which virally mediated protein expression had ended (Fig. 1b). a Superposition of paths of six rats (top). Memory is largely preserved after GFP expression but was largely erased (bottom) after expression of dominant-negative CaMKII (K42 M). b,c Summary data. A two-sample K-S test was used to determine statistical significance $(p=0.012, D=0.83 ; n=6)$. The differences on trials 1 and 7 (pre viral injection) between K42 M and GFP were not statistically significant ( $p=0.81$ and D=0.33 for trial 1; $p=0.32$ and $D=0.5$ for trial 7). Error bars represent mean \pm SEM. Asterisks indicate statistical significance ( $<<0.05$; Kolmogorov-Smirnov test). Reproduced with permission from [48] 

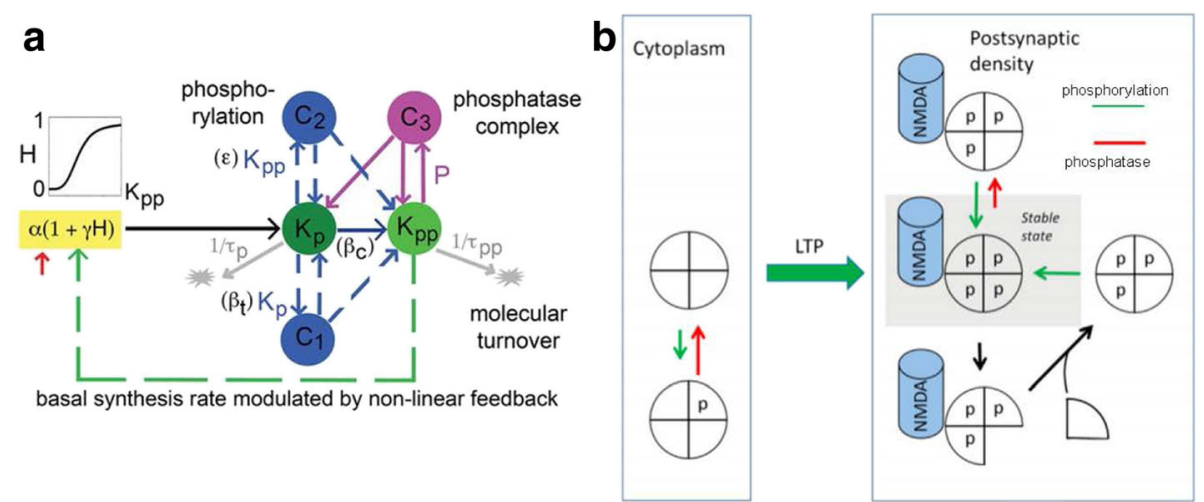

Fig. 6 Models of stable information storage by a molecular switch. a PKM-zeta model. Black arrow is protein synthesis of PKM-zeta that occurs during late phase of LTP. It is postulated that singly phosphorylated kinase (Kp) can be autophosphorylated to produce doubly phosphorylated kinase (Kpp), which then stimulates further synthesis of PKM-zeta and stable information storage. How synapse specificity is achieved is not specified. The possibility that atypical PKCs undergo such regulated phosphorylation has not been confirmed in recent work [62]. From [55]. b CaMKII model. LTP induction leads to autophosphorylation of CaMKII T286, which leads to persistent activation of the kinase and binding to the NMDA channel within the potentiated spine, thereby establishing synapse-specificity. If a subunit gets dephosphorylated (upward red arrow), the subunit is rephosphorylated by a neighboring active subunit. Protein turnover (downward black arrow) occurs by subunit exchange. A newly inserted unphosphorylated subunit will be phosphorylated by a neighboring subunit. Thus the switch will be stable despite phosphatase activity and protein turnover. From [60]

the core of both models is the concept of a positive feedback chemical system that can sustain the on-state of a switch. Because the system contains multiple molecules (subunits in the case of CaMKII) and because switch function depends on a multi-molecular system, individual molecules can be replaced by protein turnover without loss of information.

\section{Persistence of molecular modification}

LTP induction leads to persistent translocation of CaMKII to the PSD (measured $1 \mathrm{~h}$ after induction) [37] and to phosphorylation that can last for at least many hours [57]. It has not yet been possible to follow the state of CaMKII on the longer time scale. However, basal conditions measured in hippocampal slices may reflect LTP processes that occurred days before while the animal was still alive. On the simplest model, synapses start as silent (no AMPAR conductance) and LTP-like processes lead to enhancement of the AMPAR conductance. Consistent with such a model, the complex of CaMKII with the NMDAR is found under basal conditions in slices and reduction in this complex is associated with reduction in AMPAR conductance [50]. Importantly, in the presence of mutations that block CaMKII interaction with NMDAR, there is no basal AMPAR-mediated transmission [58]. A major advance would be the development of FRET methods that would allow the CaMKII bound to the NMDAR to be monitored in single spines over long periods.

In the case of PKM-zeta, recent work has monitored its learning-induced elevation over very long periods. Impressively, a $20 \%$ increase in total PKM-zeta in CA1 can be measured as long as 1 month after learning [21], making it the most persistent learning-produced biochemical change yet observed.

\section{Synapse specificity}

Given the evidence that LTP is synapse-specific (e.g., can occur at the active spine, but not at spines only a few microns away), a successful model should account for how such a high degree of localization can be achieved. In the case of CaMKII, a FRET-based reporter of activated CaMKII shows that this species is largely restricted to the stimulated spine, thus providing a local biochemical signal that can account for synapse specificity [36]. The local changes may include binding to NMDARs in that spine [41, 59], thereby forming a synapse-specific molecular engram within the postsynaptic density of the activated spine. This complex may then serve as a structural seed for the addition of other proteins, leading to trans-synaptic growth of synapse and the associated addition of AMPA channels [60].

Recent work on PKM-zeta demonstrates its role in nuclear signaling [61]. After LTP induction, PKM-zeta moves through the dendrite to the nucleus. It is active there in phosphorylating CREB binding protein (CBP). This, it is argued, might produce epigenetic changes necessary for long-term memory. However, the fact that active kinase is spreading through the dendrites to the nucleus poses a problem for any memory storage model because the active kinase could easily destroy the specific-specific action required for proper memory function. On the other hand, the spread of potentiation could contribute to a synaptic scaling function that is 
not synapse-specific. Indeed, such a role would help to account for the results of the occlusion test, which are more consistent with a role in scaling than synapsespecific memory storage.

\section{Conclusions}

The molecular basis of memory storage is one of the most fundamental questions in cellular neuroscience. It is remarkable that such a fundamental question has remained unanswered. One reason for limited progress is the difficulty of conducting the key erasure test. This test requires not only target specificity, a specificity that is difficult to achieve in vivo by traditional pharmacological methods, but also temporal control: as noted above, proper execution of the erasure test requires that the agent used to attack a putative memory molecule must be introduced and then removed. These requirements for specificity and temporal control have now been met using the HSV system for viral delivery of dominant-negative CaMKII. The results clearly demonstrate memory erasure. Similar erasure had been previously achieved in slice experiments on LTP. Thus, a reasonable conclusion is that memory is stored by an LTP-like process that depends on CaMKII.

\begin{abstract}
Abbreviations
AMPA: a-amino-3-hydroxy-5-methyl-4-isoxazolepropionic acid (receptor); CaMKII: Calcium-Calmodulin-dependent Protein Kinase Type II;

EPSP: Excitatory postsynaptic potential; HSV: Herpes simplex virus; KD: Kinase dead; Kp: Kinase phosphorylated; Kpp: Kinase doubly phosphorylated; LTP: Long-term potentiation; NMDA: N-methy-D-aspartate (receptor); PKC: Protein kinase C; PKM: Protein kinase M; PSD: Postsynaptic density; WT: Wild type
\end{abstract}

\section{Acknowledgements}

Not applicable

\section{Funding}

This work was supported by NSF IOC-1526941, NIH U01MS090583, NIH R56NS096710, NIH R01DA043195, NIH R01NS103168.

\section{Availability of data and materials}

Data sharing is not applicable to this article as no datasets were generated or analyzed in current review.

\section{Authors' contribution}

$J$ is a sole contributor to this review.

\section{Ethics approval and consent to participate}

Not applicable

\section{Consent for publication}

Not applicable

\section{Competing interests}

The author declares that he/she has no competing interests.

\section{Publisher's Note}

Springer Nature remains neutral with regard to jurisdictional claims in published maps and institutional affiliations.
Received: 20 September 2017 Accepted: 16 November 2017

Published online: 29 November 2017

\section{References}

1. Liu X, Ramirez S, Pang PT, Puryear CB, Govindarajan A, Deisseroth K, Tonegawa S. Optogenetic stimulation of a hippocampal engram activates fear memory recall. Nature. 2012:484(7394):381-5.

2. Bliss TV, Collingridge GL. A synaptic model of memory: long-term potentiation in the hippocampus. Nature. 1993:361(6407):31-9.

3. Morris RG. Long-term potentiation and memory. Philos Trans R Soc Lond Ser B Biol Sci. 2003:358(1432):643-7.

4. Brun VH, Ytterbo K, Morris RG, Moser MB, Moser El. Retrograde amnesia for spatial memory induced by NMDA receptor-mediated long-term potentiation. J Neurosci. 2001;21(1):356-62.

5. Nabavi S, Fox R, Proulx CD, Lin JY, Tsien RY, Malinow R. Engineering a memory with LTD and LTP. Nature. 2014;511(7509):348-52.

6. Whitlock JR, Heynen AJ, Shuler MG, Bear MF. Learning induces long-term potentiation in the hippocampus. Science. 2006;313(5790):1093-7.

7. Ryan TJ, Roy DS, Pignatelli M, Arons A, Tonegawa S. Memory. Engram cells retain memory under retrograde amnesia. Science. 2015;348(6238):1007-13.

8. Matsuzaki M, Honkura N, Ellis-Davies GC, Kasai H. Structural basis of longterm potentiation in single dendritic spines. Nature. 2004;429(6993):761-6.

9. Liu K, Hagan M, Lisman J: Gradation ( 10 size states) of synaptic strength by quantal addition of structural modules. Phil Trans R Soc B 2016, accepted.

10. West MJ, Gundersen HJ. Unbiased stereological estimation of the number of neurons in the human hippocampus. J Comp Neurol. 1990;296(1):1-22.

11. Kelso SR, Ganong AH, Brown TH. Hebbian synapses in hippocampus. Proc Natl Acad Sci U S A. 1986;83(14):5326-30.

12. Lisman JE. A mechanism for memory storage insensitive to molecular turnover: a bistable autophosphorylating kinase. Proc Natl Acad Sci U S A. 1985:82(9):3055-7.

13. Lisman JE, Goldring MA. Feasibility of long-term storage of graded information by the Ca2+/calmodulin-dependent protein kinase molecules of the postsynaptic density. Proc Natl Acad Sci U S A. 1988:85(14):5320-4.

14. Sacktor TC. How does PKMzeta maintain long-term memory? Nat Rev Neurosci. 2011:12(1):9-15.

15. Tsokas P, Hsieh C, Yao Y, Lesburgueres E, Wallace EJ, Tcherepanov A, Jothianandan D, Hartley BR, Pan L, Rivard B, et al. Compensation for PKMzeta in long-term potentiation and spatial long-term memory in mutant mice. elife. 2016:5:e14846 doi:10.7554/eLife.14846.

16. Si K, Kandel ER. The role of functional Prion-like proteins in the persistence of memory. Cold Spring Harb Perspect Biol. 2016;8(4):a021774.

17. Tsien RY. Very long-term memories may be stored in the pattern of holes in the perineuronal net. Proc Natl Acad Sci U S A. 2013;110(30):12456-61.

18. Frey $U$, Krug M, Reymann KG, Matthies $H$. Anisomycin, an inhibitor of protein synthesis, blocks late phases of LTP phenomena in the hippocampal CA1 region in vitro. Brain Res. 1988;452(1-2):57-65.

19. Bosch M, Castro J, Saneyoshi T, Matsuno H, Sur M, Hayashi Y. Structural and molecular remodeling of dendritic spine substructures during long-term potentiation. Neuron. 2014;82(2):444-59.

20. Meyer D, Bonhoeffer T, Scheuss V. Balance and stability of synaptic structures during synaptic plasticity. Neuron. 2014;82(2):430-43.

21. Hsieh C, Tsokas P, Serrano P, Hernandez Al, Tian D, Cottrell JE, Shouval HZ, Fenton AA, Sacktor TC. Persistent increased PKMzeta in long-term and remote spatial memory. Neurobiol Learn Mem. 2016:

22. Pastalkova E, Serrano P, Pinkhasova D, Wallace E, Fenton AA, Sacktor TC. Storage of spatial information by the maintenance mechanism of LTP. Science. 2006;313(5790):1141-4

23. Lisman J. Memory erasure by very high concentrations of ZIP may not be due to PKM-zeta. Hippocampus. 2012;22(3):648-9.

24. Volk LJ, Bachman JL, Johnson R, Yu Y, Huganir RL. PKM-zeta is not required for hippocampal synaptic plasticity, learning and memory. Nature. 2013;493(7432):420-3.

25. Lee AM, Kanter BR, Wang D, Lim JP, Zou ME, Qiu C, McMahon T, Dadgar J, Fischbach-Weiss SC, Messing RO. Prkcz null mice show normal learning and memory. Nature. 2013:493(7432):416-9.

26. Shema R, Haramati S, Ron S, Hazvi S, Chen A, Sacktor TC, Dudai Y. Enhancement of consolidated long-term memory by overexpression of protein kinase Mzeta in the neocortex. Science. 2011;331(6021):1207-10. 
27. Ling DS, Benardo LS, Serrano PA, Blace N, Kelly MT, Crary JF, Sacktor TC. Protein kinase Mzeta is necessary and sufficient for LTP maintenance. Nat Neurosci. 2002;5(4):295-6.

28. Schuette SR, Fernandez-Fernandez D, Lamla T, Rosenbrock H, Hobson S. Overexpression of protein Kinase Mzeta in the hippocampus enhances long-term Potentiation and long-term contextual but not cued fear memory in rats. J Neurosci. 2016;36(15):4313-24.

29. Wu-Zhang AX, Schramm CL, Nabavi S, Malinow R, Newton AC. Cellular pharmacology of protein kinase Mzeta (PKMzeta) contrasts with its in vitro profile: implications for PKMzeta as a mediator of memory. J Biol Chem. 2012;287(16):12879-85.

30. Sadeh N, Verbitsky S, Dudai Y, Segal M. Zeta inhibitory peptide, a candidate inhibitor of protein Kinase Mzeta, is Excitotoxic to cultured Hippocampal neurons. J Neurosci. 2015;35(36):12404-11.

31. LeBlancq MJ, McKinney TL, Dickson CT, It ZIP, Neural Silencing I. An additiona effect of the PKM-zeta inhibitor zeta-inhibitory peptide. J Neurosci. 2016;36(23):6193-8.

32. Kennedy MB, Bennett MK, Erondu NE. Biochemical and immunochemical evidence that the "major postsynaptic density protein" is a subunit of a calmodulin-dependent protein kinase. Proc Natl Acad Sci U S A. 1983:80(23):7357-61.

33. Lisman J, Yasuda R, Raghavachari S. Mechanisms of CaMKIl action in long-term potentiation. Nat Rev Neurosci. 2012;13(3):169-82.

34. Miller SG, Kennedy MB. Regulation of brain type II Ca2+/calmodulin-dependent protein kinase by autophosphorylation: a Ca2+-triggered molecular switch. Cell. 1986;44(6):861-70.

35. Otmakhov N, Lisman J. Measuring CaMKII concentration in dendritic spines. J Neurosci Methods. 2012;203(1):106-14.

36. Lee SJ, Escobedo-Lozoya Y, Szatmari EM, Yasuda R. Activation of CaMKII in single dendritic spines during long-term potentiation. Nature. 2009; 458(7236):299-304

37. Otmakhov N, Tao-Cheng JH, Carpenter S, Asrican B, Dosemeci A, Reese TS, Lisman J. Persistent accumulation of calcium/calmodulin-dependent protein kinase II in dendritic spines after induction of NMDA receptor-dependent chemical long-term potentiation. J Neurosci. 2004;24(42):9324-31.

38. Dosemeci A, Weinberg RJ, Reese TS, Tao-Cheng JH. The postsynaptic density: there is more than meets the eye. Front Synaptic Neurosci. 2016;8:23.

39. Petersen JD, Chen X, Vinade L, Dosemeci A, Lisman JE, Reese TS. Distribution of postsynaptic density (PSD)-95 and Ca2+/calmodulin-dependent protein kinase II at the PSD. J Neurosci. 2003:23(35):11270-8.

40. Bayer KU, De Koninck P, Leonard AS, Hell JW, Schulman H. Interaction with the NMDA receptor locks CaMKII in an active conformation. Nature. 2001;411(6839):801-5.

41. Strack S, Colbran RJ. Autophosphorylation-dependent targeting of calcium/ calmodulin-dependent protein kinase II by the NR2B subunit of the $\mathrm{N}$ methyl- D-aspartate receptor. J Biol Chem. 1998;273(33):20689-92.

42. Barria A, Malinow R. NMDA receptor subunit composition controls synaptic plasticity by regulating binding to CaMKII. Neuron. 2005:48(2):289-301.

43. Silva AJ, Paylor R, Wehner JM, Tonegawa S. Impaired spatial learning in alphacalcium-calmodulin kinase II mutant mice. Science. 1992;257(5067):206-11.

44. Giese KP, Fedorov NB, Filipkowski RK, Silva AJ. Autophosphorylation at Thr286 of the alpha calcium-calmodulin kinase II in LTP and learning. Science. 1998:279(5352):870-3

45. Yamagata Y, Kobayashi S, Umeda T, Inoue A, Sakagami H, Fukaya M, Watanabe M, Hatanaka N, Totsuka M, Yagi T, et al. Kinase-dead knock-in mouse reveals an essential role of kinase activity of Ca2+/calmodulin-dependent protein kinase llalpha in dendritic spine enlargement, long-term potentiation, and learning. J Neurosci. 2009;29(23):7607-18.

46. Lledo PM, Hjelmstad GO, Mukherji S, Soderling TR, Malenka RC, Nicoll RA. Calcium/calmodulin-dependent kinase II and long-term potentiation enhance synaptic transmission by the same mechanism. Proc Natl Acad Sci U S A. 1995;92(24):11175-9.

47. Pi HJ, Otmakhov N, Lemelin D, De Koninck P, Lisman J. Autonomous CaMKII can promote either long-term potentiation or long-term depression, depending on the state of T305/T306 phosphorylation. J Neurosci. 2010;30(26):8704-9.

48. Rossetti T, Banerjee S, Kim C, Leubner M, Lamar C, Gupta P, Lee B, Neve R, Lisman J: Memory erasure experiments indicate a critical role of CaMKII in memory storage. Neuron 2017, In Print.

49. Vest RS, Davies KD, O'Leary H, Port JD, Bayer KU. Dual mechanism of a natural CaMKII inhibitor. Mol Biol Cell. 2007:18(12):5024-33.
50. Sanhueza M, Fernandez-Villalobos G, Stein IS, Kasumova G, Zhang P, Bayer KU, Otmakhov N, Hell JW, Lisman J. Role of the CaMKII/NMDA receptor complex in the maintenance of synaptic strength. J Neurosci. 2011;31(25): 9170-8.

51. Gouet C, Aburto B, Vergara C, Sanhueza M. On the mechanism of synaptic depression induced by CaMKIIN, an endogenous inhibitor of CaMKII. PLoS One. 2012;7(11):e49293.

52. Barcomb K, Hell JW, Benke TA, Bayer KU. The CaMKII/GluN2B protein interaction maintains synaptic strength. J Biol Chem. 2016;291(31):16082-9.

53. Carlezon WA, Jr., Boundy VA, Haile CN, Lane SB, Kalb RG, Neve RL, Nestler EJ: Sensitization to morphine induced by viral-mediated gene transfer. Science 1997, 277(5327):812-814

54. Alvarez-Castelao B, Schuman EM. The regulation of synaptic protein turnover. J Biol Chem. 2015:290(48):28623-30.

55. Jalil SJ, Sacktor TC, Shouval HZ. Atypical PKCs in memory maintenance: the roles of feedback and redundancy. Learn Mem. 2015;22(7):344-53.

56. Miller P, Zhabotinsky AM, Lisman JE, Wang XJ. The stability of a stochastic CaMKII switch: dependence on the number of enzyme molecules and protein turnover. PLoS Biol. 2005;3(4):e107.

57. Ahmed T, Frey JU. Plasticity-specific phosphorylation of CaMKII, MAP-kinases and CREB during late-LTP in rat hippocampal slices in vitro. Neuropharmacology. 2005;49(4):477-92

58. Incontro S, Asensio C, Nicoll RA. A novel structural role for calcium/ calmodulin kinase II in hippocampal pyramidal neurons. In: Society for Neurosciences Annual Meeting. San Diego: Society for Neurosciences. 2016. SFN:685.607/G630

59. Leonard AS, Lim IA, Hemsworth DE, Horne MC, Hell JW. Calcium/ calmodulin-dependent protein kinase $\|$ is associated with the N-methyl-Daspartate receptor. Proc Natl Acad Sci U S A. 1999;96(6):3239-44.

60. Lisman J, Raghavachari S. Biochemical principles underlying the stable maintenance of LTP by the CaMKII/NMDAR complex. Brain Res. 2015;1621:51-61.

61. Ko HG, Kim Jl, Sim SE, Kim T, Yoo J, Choi SL, Baek SH, WJ Y, Yoon JB, Sacktor $\mathrm{TC}$, et al. The role of nuclear PKMzeta in memory maintenance. Neurobiol Learn Mem. 2016:135:50-6.

62. Tobias IS, Kaulich M, Kim PK, Simon N, Jacinto E, Dowdy SF, King CC, Newton AC. Protein kinase Czeta exhibits constitutive phosphorylation and phosphatidylinositol-3,4,5-triphosphate-independent regulation. Biochem J. 2016:473(4):509-23.

\section{Submit your next manuscript to BioMed Central and we will help you at every step:}

- We accept pre-submission inquiries

- Our selector tool helps you to find the most relevant journal

- We provide round the clock customer support

- Convenient online submission

- Thorough peer review

- Inclusion in PubMed and all major indexing services

- Maximum visibility for your research

Submit your manuscript at www.biomedcentral.com/submit 